\title{
Multiplicity of Approach and Method in Augmentation of Simplex Method: A Review
}

\author{
Nor Asmaa Alyaa Nor Azlan ${ }^{1}$, Effendi Mohamad ${ }^{1, *}$, Mohd Rizal Salleh ${ }^{1}$, Oyong Novareza ${ }^{2}$, \\ Dani Yuniawan ${ }^{3}$, Muhamad Arfauz A Rahman', Adi Saptari ${ }^{4}$, Mohd Amri Sulaiman ${ }^{1}$ \\ ${ }^{1}$ Faculty of Manufacturing Engineering, Universiti Teknikal Malaysia Melaka (UTeM), Malaysia \\ ${ }^{2}$ Department of Industrial Engineering, Brawijya University, Indonesia \\ ${ }^{3}$ Department of Industrial Engineering, Merdeka Malang University, Indonesia \\ ${ }^{4}$ Department of Industrial Engineering, President University, Indonesia
}

Received June 17, 2019; Revised October 1, 2019; Accepted December 23, 2019

Copyright $\subseteq 2020$ by authors, all rights reserved. Authors agree that this article remains permanently open access under the terms of the Creative Commons Attribution License 4.0 International License

\begin{abstract}
The purpose of this review paper is to set an augmentation approach and exemplify distribution of augmentation works in Simplex method. The augmentation approach is classified into three forms whereby it comprises addition, substitution and integration. From the diversity study, the result shows that substitution approach appeared to be the highest usage frequency, which is about $45.2 \%$ from the total of percentage. This is then followed by addition approach which makes up $32.3 \%$ of usage frequency and integration approach for about $22.6 \%$ of usage frequency which makes it the least percentage of the overall usage frequency approach. Since it is being the least usage percentage, the paper is then interested to foresee a future study of integration approach that can be performed from the executed distribution of the augmentation works according to Simplex's computation stages. A theme screening is then conducted with a set of criteria and themes to come out with a proposal of new integration approach of augmentation of Simplex method.
\end{abstract}

Keywords Simplex Method, Augmentation of Simplex Method, Multiplicity of Approach

\section{Introduction}

Simplex algorithm is an interesting method solving linear programming (LP) problem which is cited in extensive areas of knowledge and abundance numbers of publications versus years [1]. Plus, the most essential merits are the algorithm that is very useful in multiple dimensional optimization problems, robust technique, firm local optimization and easy to be applied numerically [2]. However, the only drawback of Simplex algorithm is involving its computation pitfall in initialization, iteration calculation and termination stage [3-5]. Due to this circumstance, this has given opportunities to other researchers to do augmentation of Simplex algorithm in many ways.

\section{Objective}

It is acknowledged that, a lot of well-known augmentation works have improvised the algorithm. The improvements are whichever desires to enhance the computation stages whereby the initialization, iterative calculation and termination of the algorithm [6]. Yet, the augmentation works can either be a modification or alteration to the algorithm and solely subjected to enhance the computation performance of Simplex method [7]. Hence, this paper is interested in reviewing on the related manuscripts in order to identify the distribution of their contribution to the computation performance enhancement and the diversity of the augmentation approach.

\section{Methods}

\subsection{Diversity of Augmentation Approach}

The augmentation works of Simplex method were developed to generate more interesting algorithm towards solving the LP problem in order to achieve optimal solution [8]. As depicted in Fig. 1, the augmentation works come with different kinds of approaches applied in solving LP problem using the Simplex algorithm as its core. The diversity of the augmentation approaches can be divided into three types of modification which are addition, 
substitution and integration [9-11]. The addition is when the modification is done by introducing new or other existing methods into the algorithm of conventional Simplex method [12].

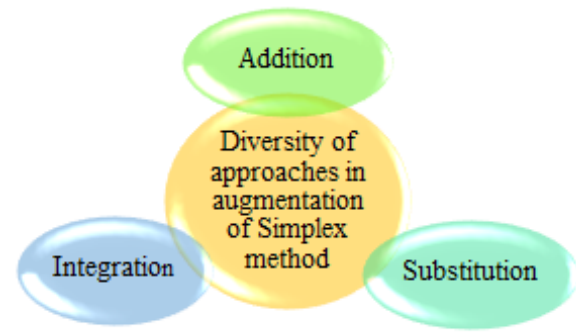

Figure 1. Diversity of augmentation approach [9-11]

Table 1. Classification existed augmentation works of Simplex algorithm approach

\begin{tabular}{|c|c|c|}
\hline \multirow{4}{*}{ 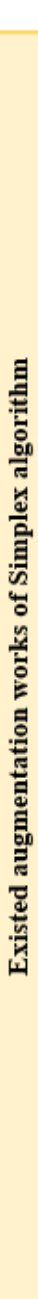 } & & Augmentation approach \\
\hline & 胥 & $\begin{array}{l}\text { - Three-phase Simplex type solution algorithm } \\
\text { - }\end{array}$ \\
\hline & 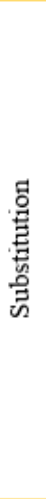 & 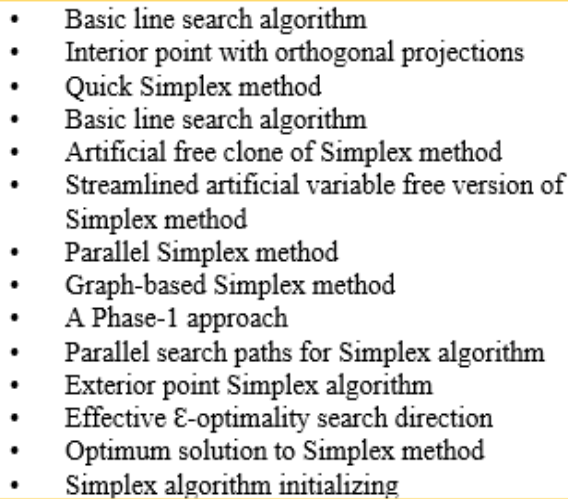 \\
\hline & 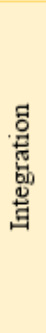 & $\begin{array}{l}\text { - Network Simplex method } \\
\text { - } \text { Revised multi-phase Simplex Algorithm } \\
\text { method lightning search algorithm -Simplex } \\
\text { - Hybrid genetic algorithm with Simplex } \\
\text { method } \\
\text { - Simplex method-based social spider } \\
\text { optimization algorithm } \\
\text { - } \\
\text { Augmented downhill Simplex method } \\
\text { Generalized rotation-symmetry-model }\end{array}$ \\
\hline
\end{tabular}

Whereas the substitution type of modification is when there are changes in the algorithm of conventional Simplex method and it is then replaced with new or other existing methods[13]. While the integration type of modification is a combination of at least two existing methods to form a new augmentation of Simplex method [14]. These three types of augmentation approaches can be seen in their works by gathering and classifying the existing augmentation works under these approaches as tabulated in Table 1. These existing augmentation works of Simplex method were limited to works that focused on the LP problems with linear objective function and constraints.

\subsection{Arguments on Diversity of Augmentation Approach}

As tabulated in Table 1, there are varieties of numbers of augmentation works for past five years of 2018, for each augmentation approach resulted from this classification. In order to see the trend, a line chart is plotted as portrayed in Fig. 2. The plotted line chart shows percentage of each augmentation approach applied in existed augmentation works for the past five years of 2018 and those years older than the past five years of 2018 which is older than year of 2013.

The higher percentage indicates the augmentation approach has been frequently applied as well as abundantly found in literatures and so vice versa. By looking at those years older than year of 2013, both addition and substitution approaches have shown highest percentage of $47.1 \%$ of usage frequency whereas integration has recorded lowest percentage whereby about $3.0 \%$ only. This signifies the addition and substitution approaches that are more notorious compared to the integration approach on augmenting Simplex algorithm during those years older than year 2013 [6-7,15-19].

Moving on to the augmentation works during the year of 2013, integration approach experienced a sudden spike of usage frequency for about $50.0 \%$. The same percentage figure was also being shared with substitution approach that experienced a slight increment from previous years. This reflects that the integration approach has begun to gain interest among the researchers to apply this approach in their augmentation works of Simplex method [14]. Yet, substitution approach is continuously being the preferred approach on the augmentation studies [20]. However, addition approach has declined dramatically and this situation persists until year of 2015. 


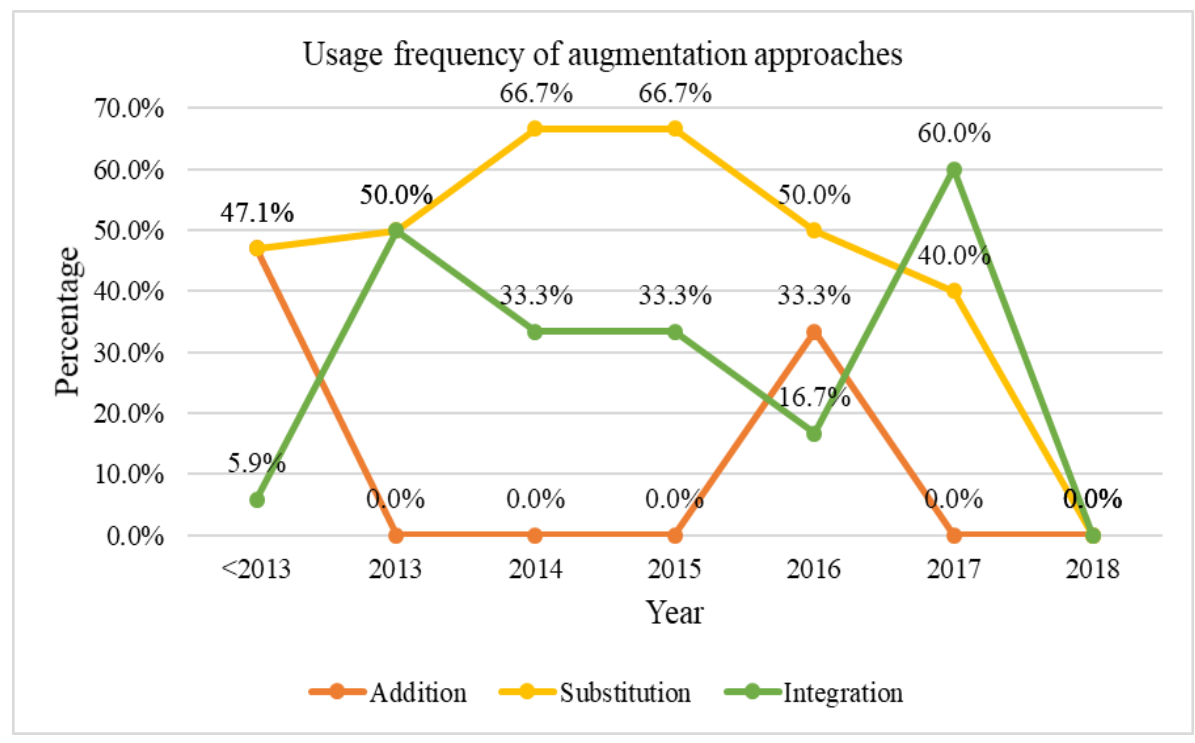

Figure 2. Percentage of usage frequency of augmentation approach

As for the integration, this approach underwent a marginal decrease in the percentage of usage frequency whereby from $50.0 \%$ to $33.3 \%$ for the year of 2013 to 2014 respectively and the percentage of usage frequency maintained until the year 2015. Even though there is declining in the studies using integration approach, but the works are steadily relevant among the researchers $[9,21]$. While for substitution approach, it is consistently to incline in the percentage of usage frequency for the year of 2014 which is $66.7 \%$ and this percentage is remained until the year of 2015. This positive progress shows that substitution is the most actively approach that is used by the researchers compared to the other two approaches during the year of 2015 [11,22-24].

Nevertheless, in 2016, both substitution and integration approaches experience a percentage recession of usage frequency whereby both are declined to $50.0 \%$ and $16.7 \%$ respectively from the previous year. Still, these two approaches managed to sustain their existence in contributing to the augmentation works of Simplex algorithm [25-26]. Nonetheless, the addition approach was inclined drastically in the year 2016 for about $50.0 \%$ of the usage frequency. This reflects that the researchers may have invented new methods to be applied into the algorithm of Simplex by the addition approach and eventually contributed to the augmentation studies [27]. Though, this condition didn't last long as the literatures on this approach declined in the year of 2017.

The substitution approach experienced year by year declination of usage percentage until $40.0 \%$ in the year of
2017. In spite of that, the integration approach was managed to get back in ascending behaviour in the year of 2017 whereby it collects the percentage of usage frequency up to $60.0 \%$. These situations indicate that the researchers were most likely to apply integration as their approach to augment the algorithm as they may find that this approach was very convenient to be worked with and offer more chances to generate new algorithm of Simplex method $[2,13,28-29]$.

\subsection{Distribution of Augmentation Works}

The extensions of Simplex method in augmenting the algorithm were derived from the issue of pitfalls in the computation stages of Simplex. It is said so as the pitfalls that were experienced by users during performing the computation have influenced and led the researchers to put efforts on inventing alternative methods to improvise the algorithm. The invented alternative methods were none other than referring to the augmentation works to enhance the initialization, iterative calculation and termination stage.

Hence, the distribution of these augmentation works of Simplex will be clarified according to these three computation stages of the algorithm. Based on Table 2, there are about 53 augmentation works that were applied in augmenting the Simplex algorithm through the computation stages whereby each initialization, iterative calculation and termination were being applied about 30 methods, 10 methods and 13 methods respectively. 
Table 2. Classification existed augmentation works of Simplex algorithm approach

\begin{tabular}{|c|c|c|c|}
\hline & & \multicolumn{2}{|r|}{ Existed augmentation works of Simplex algorithm } \\
\hline & 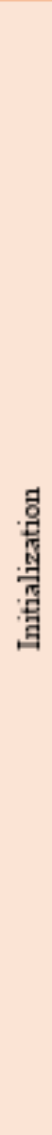 & $\begin{array}{l}1 . \\
2 . \\
3 . \\
4 . \\
5 . \\
6 . \\
7 . \\
8 . \\
9 . \\
10 \\
11 \\
12 \\
13 \\
14 \\
15 \\
16 \\
17 \\
18 \\
19 \\
20 \\
21 \\
22 \\
23 \\
24 \\
25 \\
26 \\
27 \\
28 \\
29 \\
30\end{array}$ & $\begin{array}{l}\text { Basic line search algorithm } \\
\text { Basic line search algorithm } \\
\text { Quick Simplex method } \\
\text { Three-phase simplex type solution algorithm } \\
\text { Fuzzy primal Simplex algorithm } \\
\text { Novel tabular form of Simplex method } \\
\text { Effective E-optimality search direction } \\
\text { Primal deficient-basis Simplex algorithm } \\
\text { Interval Simplex algorithm } \\
\text { BMS interval Simplex algorithm } \\
\text { Simplex algorithm extended to piecewise-linearly constrained } \\
\text { problems } \\
\text { Simplex method meeting conjectured Hirsch bound } \\
\text { New optimal pivot rule for Simplex algorithm } \\
\text { Interior point with orthogonal projections } \\
\text { Artificial free clone of Simplex method } \\
\text { Streamlined artificial variable free version of Simplex method } \\
\text { Parallel Simplex method } \\
\text { Graph-based Simplex method } \\
\text { Parallel gearch paths for Simplex algorithm } \\
\text { Exterior point Simplex algorithm } \\
\text { Alternative approach of optimum solution } \\
\text { Simplex Algorithm Initializing } \\
\text { Network Simplex method } \\
\text { Revised Multi-Phase Simplex Algorithm } \\
\text { Hybrid lightning search algorithm -Simplex method } \\
\text { Hybrid genetic algorithm with Simplex method } \\
\text { Simplex method-based social spider optimization algorithm } \\
\text { Augmented downhill Simplex } \\
\text { Generalized rotation-symmetry-model } \\
\text { A Phase-l approach }\end{array}$ \\
\hline & 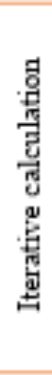 & $\begin{array}{l}1 . \\
2 . \\
3 . \\
4 . \\
5 . \\
6 . \\
7 . \\
8 . \\
9 . \\
10\end{array}$ & $\begin{array}{l}\text { Quick Simplex method } \\
\text { Interior point with orthogonal projections } \\
\text { Graph-based Simplex method } \\
\text { Network Simplex method } \\
\text { Augmented downhill Simplex } \\
\text { Generalized rotation-symmetry-model } \\
\text { Revised Multi-Phase Simplex Algorithm } \\
\text { Hybrid lightning search algorithm -Simplex } \\
\text { Hybrid genetic algorithm with Simplex method } \\
\text { Simplex method-based social spider optimization algorithm }\end{array}$ \\
\hline & 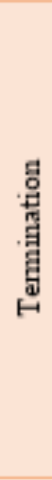 & $\begin{array}{l}1 . \\
2 . \\
3 . \\
4 . \\
5 . \\
6 . \\
7 . \\
8 . \\
9 . \\
10 \\
11 \\
12 \\
13\end{array}$ & $\begin{array}{l}\text { Basic line search algorithm } \\
\text { Basic line search algorithm } \\
\text { Generalized reviged simplex algorithm } \\
\text { Interior point with orthogonal projections } \\
\text { Graph-based Simplex method } \\
\text { Exterior point Simplex algorithm } \\
\text { Network Simplex method } \\
\text { Hybrid lightning search algorithm -Simplex method } \\
\text { Hybrid genetic algorithm with Simplex method } \\
\text { Simplex method-based social spider optimization algorithm } \\
\text { Augmented downhill Simplex } \\
\text { Generalized rotation-symmetry-model } \\
\text { Revised Multi-phase Simplex Algorithm }\end{array}$ \\
\hline
\end{tabular}




\subsection{Theme Screening Process}

Considering the integration is the least approach applied, thus, this paper plans to foresee an idea of augmentation work, which is possibly conducted by using the integration approach. Also, this is to contribute to the number of integration approach in the augmentation works of Simplex method. From all of these methods, at least two current augmentation works to be salvaged for augmenting Simplex method using integration as its type of modification. This can be achieved via a theme screening process to evaluate the method's capability towards fulfilling the study requirements.

The aim of theme screening is on assessing criteria and themes in relative terms and the assessment will be judged based on the score whereby ' + ' if the theme is better , ' 0 ' if the theme is equal and '-' if the theme is worse . In the case of this research, the 'better ' term indicates that the algorithm of Simplex will experience positive impact from the superiority offered by the augmentation works. While the 'equal ' term indicates the algorithm will experience none impact from the superiority offered by the augmentation works as the output is equal. Whereas, the 'worse' term indicates that the algorithm will experience negative impact from the superiority offered by the augmentation works.

For the criteria, efficiency and reliability of computation performance will be used as the parameters, since these extensions of Simplex method were derived from the pitfalls in the computation stages. The efficiency criterion will be broken down into the reduction of computation time and reduction of iteration time while the reliability criterion will be screened based on the increment of optimal solution accuracy and reduction of optimal solution variance. Yet, the theme will be the list of current augmentation works of Simplex method, whereby these themes will be assessed according to the decided criteria.

\subsection{Secondary Theme Screening Process}

Secondary theme screening process is required to evaluate the two remaining methods' capability towards fulfilling the study limitations. This time, the aim is on assessing criteria and themes in relative terms and the assessment will be judged based on the score whereby '+' if the theme is high impact, ' 0 ' if the theme is neutral and ' - ' if the theme is low impact. In the case of this research, the 'high impact' term indicates that the augmentation work is mainly complying on the study limitation. While the 'neutral' term indicates the study limitation is not in the purpose of the augmentation work.

Whereas, the 'low impact' term indicates that the augmentation work is not mainly complying on the study limitation. As for the criteria, study limitations will be used as the parameters, which will be screened based on the objective function and constraints whereby both must be in linear and integer form of that studied by the two augmentation works. Yet, the theme will be the basic line search algorithm and interior point with orthogonal projections that will be assessed according to the new set of criteria.

Overall usage frequency of the augmentation approach

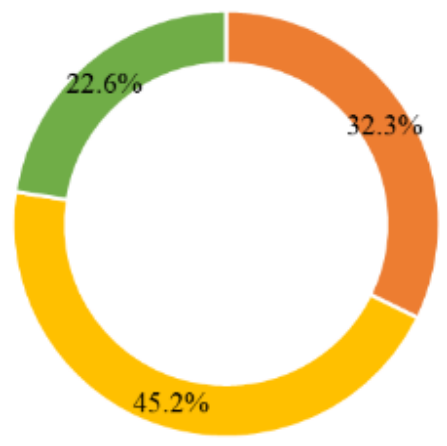

- Addition " Substitution = Integration

Figure 3. Percentage of overall usage frequency of augmentation of approach 


\section{Results}

\subsection{Result of Arguments on Diversity of Augmentation Approach}

Based on pie chart of Fig. 3, from the overall usage frequency for augmentation approach from the year of less than 2013 until the year of 2018, it can be said that the substitution approach has been the most applied approach. The substitution approach has covered about $45.2 \%$ of usage frequency from the total of percentage. As for the addition, this approach has dominated about $32.3 \%$, which makes it the next most applied approach after the substitution approach.

Whereas, the least percentage of the overall usage frequency approach is the integration whereby it covered about $22.6 \%$. From the result of this pie chart, it shows that there is an immense difference of percentage between these three approaches, which substitution has dominated almost half of the overall percentage, when compared to the addition and integration approach. As for the integration approach, it appeared to be the least approach applied in the augmentation studies. Hence, this indicates that there is a necessity to propose a new augmentation work of Simplex using the integration approach as its type of modification.

\subsection{Result of Theme Screening Process}

Referring to Table 3, this theme screening has come out with a result showing that there are three augmentation works that were managed toappear as the highest rank among all and with same total score of 3 . The three augmentation works are involving quick Simplex method, basic line search algorithm and interior point with orthogonal projections. For quick Simplex method, the result has showed the application of this augmentation work into Simplex algorithm that will give betterment on both reliability criteria.

This reflects the quick Simplex method able to increase the optimal solution accuracy as well as reduce the optimal solution variance. However, for efficiency, only one criterion will gain betterment from the augmentation work instead of both criteria. Meaning, the quick Simplex method is able to reduce the computation time but the iteration number would be at least equal to the conventional Simplex method.

Table 3. Theme screening of efficiency and reliability

\begin{tabular}{|c|c|c|c|c|c|c|}
\hline \multirow[b]{2}{*}{ Criteria } & \multicolumn{2}{|c|}{ Efficiency } & \multicolumn{2}{|c|}{ Reliability } & \multirow{2}{*}{ 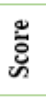 } & \multirow{2}{*}{ 当 } \\
\hline & $\begin{array}{c}\text { Less computation } \\
\text { time }\end{array}$ & $\begin{array}{c}\text { Less } \\
\text { iteration }\end{array}$ & $\begin{array}{c}\text { High optimal } \\
\text { solution accuracy }\end{array}$ & $\begin{array}{c}\text { Less optimal } \\
\text { solution variance }\end{array}$ & & \\
\hline \multicolumn{7}{|l|}{ Theme } \\
\hline Alternative optimum solution & + & + & 0 & 0 & 2 & 2 \\
\hline Graph-based Simplex & + & + & 0 & 0 & 2 & 2 \\
\hline 3-phase Simplex & - & + & 0 & 0 & 0 & 4 \\
\hline BMS interval Simplex & 0 & 0 & 0 & 0 & 0 & 4 \\
\hline Hybrid lightning search & + & - & + & + & 2 & 2 \\
\hline Interval Simplex algorithm & 0 & 0 & 0 & 0 & 0 & 4 \\
\hline Primal deficient-basis & + & + & 0 & 0 & 2 & 2 \\
\hline Efficient-optimality search direction & - & + & + & 0 & 1 & 3 \\
\hline Tabular form of Simplex & + & - & 0 & 0 & 0 & 4 \\
\hline Generalized rotation-symmetry & 0 & 0 & 0 & 0 & 0 & 4 \\
\hline Phase-1 approach & - & + & 0 & 0 & 0 & 4 \\
\hline Augmented downhill Simplex & + & - & + & + & 2 & 2 \\
\hline Social spider optimization & + & - & + & + & 2 & 2 \\
\hline Conjectured Hirsch bound & - & + & 0 & 0 & 0 & 4 \\
\hline Quick Simplex method & + & 0 & + & + & 3 & 1 \\
\hline Revised multi-phase & - & + & 0 & 0 & 0 & 4 \\
\hline Network Simplex method & - & 0 & 0 & 0 & -1 & 5 \\
\hline Simplex algorithm initializing & + & + & 0 & 0 & 2 & 2 \\
\hline Basic line search algorithm & 0 & + & + & + & 3 & 1 \\
\hline Exterior point Simplex & - & + & 0 & 0 & 0 & 4 \\
\hline Parallel search paths & + & + & 0 & 0 & 2 & 2 \\
\hline Fuzzy primal Simplex & - & - & 0 & 0 & -2 & 6 \\
\hline Parallel Simplex method & 0 & - & 0 & 0 & -1 & 5 \\
\hline Streamlined artificial variable & + & + & 0 & 0 & 2 & 2 \\
\hline Artificial free clone & + & + & 0 & 0 & 2 & 2 \\
\hline Interior point with orthogonal projections & 0 & + & + & + & 3 & 1 \\
\hline New optimal pivot rule & + & + & 0 & 0 & 2 & 2 \\
\hline Hybrid genetic algorithm & 0 & 0 & + & + & 2 & 2 \\
\hline Piecewise-linearly constrained & + & - & 0 & 0 & 0 & 4 \\
\hline
\end{tabular}


For the basic line search algorithm and interior point with orthogonal projections, the result has showed that these two augmentation works having the same scoring upshot. The application of these augmentation works into Simplex algorithm will give betterment on both reliability criteria. This reveals, the two augmentation works are able to increase the optimal solution accuracy as well as reduce the optimal solution variance.

However, for efficiency, only one criterion will gain betterment from those two augmentation works instead of both criteria. Meaning, those two augmentation works are able to reduce the iteration number but the computation time would be at least equal to the conventional Simplex method.

\subsection{Result of Secondary Theme Screening Process}

Referring to Table 4, as the score was given to each augmentation work and then ranked according to their score, this theme screening has come out with a result showing that basic line search algorithm managed to accumulate higher score than the interior point with orthogonal projections whereby each scoring of 4 and 3 respectively.

Table 4. Theme screening of study scope and limitation

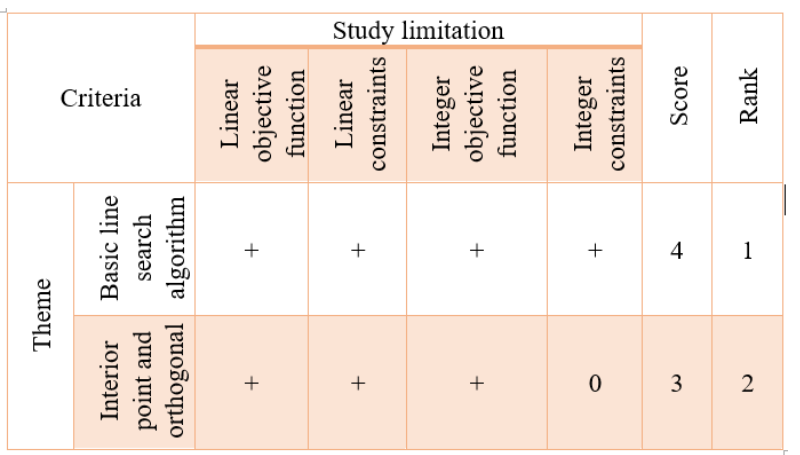

\section{Discussion}

In summary, all the three augmentation works have surpassed both of the reliability's criteria but not the efficiency's criteria. One of the efficiency's criteria which is the reduction of computation time is solitary surpassed by the quick Simplex method. While the reduction of iteration number is surpassed by the other two works which are basic line search algorithm and interior point with orthogonal projections.

Considering this circumstance, in order to have both efficiency's criteria to be surpassed, the quick Simplex method apparently would be selected as one of the two augmentation works to be integrated with one of those two works. Thus, a fine filtering needed to decide which among of the two augmentation works is selected as to be integrated with the quick Simplex method later on.

For basic line search algorithm, the theme screening result has showed this augmentation work is capable to fully commit all the set criteria. Yet, for the interior point with orthogonal projections, the augmentation work managed to commit only three out of the four set criteria. This is because the study limitation of integer constraints criteria is not in the purpose of the augmentation work.

Therefore, basic line search algorithm will be selected as the augmentation works to be possibly integrated with the quick Simplex method to generate a new augmentation algorithm, as illustrated in Fig. 4.

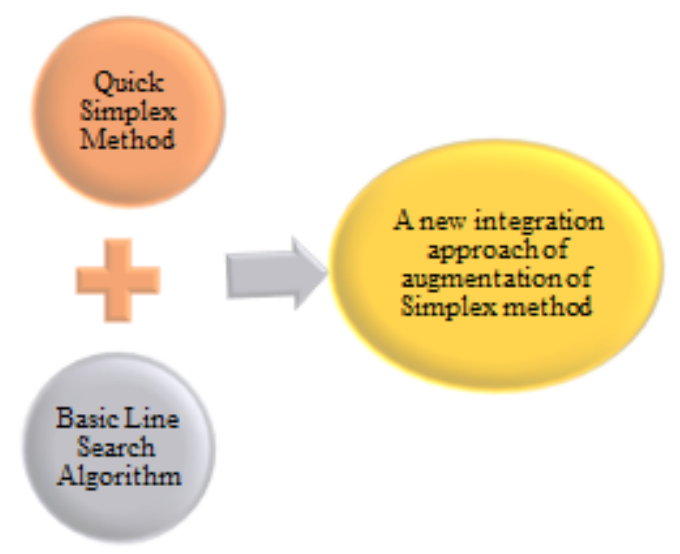

Figure 4. New proposed method of augmenting Simplex using integration approach

\section{Conclusions}

In summary, it is coveted that other related researchers or academicians to contribute more exploration to the augmentation works of Simplex method using the integration approach as its current works are only less than half (about 22.6\%) of the overall methods for solving LP problems available. In addition to that, there are masses of extended methods and algorithms available that can be exploited using the integration approach to hybrid their offered superiors which eventually will generate more interesting algorithm improving the existing deficiencies with better efficiency as well as reliability result.

\section{Acknowledgements}

The authors would like to thank the Malaysian Government, Universiti Teknikal Malaysia Melaka (UTeM) and Fakulti Kejuruteraan Pembuatan for their support and cooperation.

\section{REFERENCES}

[1] N. Ploskas and N. Samaras, "Pivoting rules for the revised 
simplex algorithm,” Yugosl. J. Oper. Res., vol. 24, no. 3, pp. 321-332, 2014.

[2] S. Voulgaropoulou, N. Samaras, and A. Sifaleras, "Computational complexity of the exterior point simplex algorithm,” Oper. Res., no. November, pp. 1-20, 2017.

[3] N. A. A. N. Azlan, A. Saptari, and E. Mohamad, "Augmentation of Simplex Algorithm for Linear Programming Problem to Enhance Computational Performance,” J. Adv. Manuf. Technol. -Special Issue Symp. 2017, vol. 11, no. November, pp. 31-46, 2017.

[4] J. Xu, E. Huang, L. Hsieh, L. H. Lee, Q. S. Jia, and C. H. Chen, "Simulation optimization in the era of Industrial 4.0 and the Industrial Internet,” J. Simul., vol. 10, no. 4, pp. 310-320, 2016.

[5] M. Holzhauser, S. O. Krumke, and C. Thielen, "On the complexity and approximability of budget-constrained minimum cost flows,” Inf. Process. Lett., vol. 126, pp. 2429, 2017.

[6] S. Zhu, G. Ruan, and X. Huang, "Some fundamental issues of basic line search algorithm for linear programming problems,” Optimization, vol. 59, no. 8, pp. 1283-1295, 2010.

[7] N. V Vaidya and N. W. Khobragade, "Optimum Solution to the Simplex Method - An Alternative Approach,” Int. J. Latest Trends Math., vol. 2, no. 3, pp. 99-105, 2012.

[8] M. Jalaeian-f, “Augmented Downhill Simplex a Modified Heuristic Optimization Method,” J. Comput. Robot., vol. 5, no. 2, pp. 1-6, 2012.

[9] M. Gohl and K. H. Borgwardt, "The Average Number of Pivot Steps of the Simplex-Algorithm Based on a Generalized Rotation-Symmetry-Model,” Math. METHODS Oper. Res., vol. 80, no. 3, pp. 329-366, 2014.

[10] A. Obot, U. M. Anthony, and S. Ozuomba, “A Novel Tabular Form of the Simplex Method for Solving Linear Programming Problems,” Int. J. Comput. Sci. Netw. Solut., vol. 4, no. 2, pp. 1-8, 2016.

[11] D. Prüša, "Graph-based simplex method for pairwise energy minimization with binary variables," Proc. IEEE Comput. Soc. Conf. Comput. Vis. Pattern Recognit., vol. 07-12-June, no. April, pp. 475-483, 2015.

[12] H. Suprajitno and I. Mohd, "Linear Programming with Interval Arithmetic,” Science (80-. )., vol. 5, no. 7, pp. 323332, 2010.

[13] P. Tar, B. Stagel, and I. Maros, "Parallel search paths for the Simplex algorithm,” Cent. Eur. J. Oper. Res., vol. 25, no. 4, pp. 967-984, 2017.

[14] E. Eroglu, “An Application of Network Simplex Method for Minimum Cost Flow Problems,” Balk. J. Math., vol. 01, no. January, pp. 117-130, 2013.

[15] G. Karypis and V. Kumar, "Performance and Scalability of the Parallel Simplex Method for Dense Linear Programming Problems An Extended Abstract,” no. MAY, 1994.

[16] H. Luh and R. Tsaih, "An efficient search direction for linear programming problems,” Comput. Oper. Res., vol. 29, no. 2, pp. 195-203, 2002.
[17] P. Pan, "A Phase-1 Approach for the Generalized Simplex Algorithm,” PERGAMON Comput. Math. with Appl. 42, vol. 42, pp. 1455-1464, 2001.

[18] A. L. Ramírez, O. Buitrago, R. A. Britto, and A. Fedossova, "A new algorithm for solving linear programming problems,” Ing. e Investig., vol. 32, no. 2, pp. 68-73, 2012.

[19] N. V. Stojković, P. S. Stanimirović, M. D. Petković, and D. S. Milojković, "On the simplex algorithm initializing," Abstr. Appl. Anal., vol. 2012, no. November, pp. 1-15, 2012.

[20] M. Imtiaz, N. Touheed, and S. Inayatullah, "Artificial Free Clone of Simplex Method for Feasibility,” Publ. SIAM Rev., pp. 1-17, 2013.

[21] F. Wang, C. Wang, Z. Wang, and X. Zhang, “A hybrid algorithm of GA + simplex method in the WSN localization,” Int. J. Distrib. Sens. Networks, vol. 2015, 2015.

[22] M. N. Dumaldar, "A theoretical comparison between the Simplex method and the asic line search algorithm," Optimization, vol. 65, no. 1, pp. 1-9, 2014.

[23] S. Inayatullah, N. Touheed, and M. Imtiaz, “A streamlined artificial variable free version of simplex method," Public Libr. Sci. ONE, vol. 10, no. 3, pp. 1-28, 2015.

[24] N. V. Vaidya and N. N. Kasturiwale, "Quick Simplex Algorithm for Optimal Solution to the Linear Programming Problem along with Theoretical Proof of ... Quick Simplex Algorithm for Optimal Solution to the Linear Programming Problem along with Theoretical Proof of Formulae .,” Int. J. Latest Trend Math., vol. 4, no. 2, pp. 183-200, 2014.

[25] G. T. Mohammed and B. G. Hordofa, "The Modified Sequential Linear Goal Programming Method for Solving Multiple Objectives Linear Programming Problems,” Pure Appl. Math. J., vol. 5, no. 1, pp. 1-8, 2016.

[26] N. Vaidya and N. Kasturiwale, “Application of Quick Simplex Method (A New Approach) On Two Phase Method,” Br. J. Math. Comput. Sci., vol. 16, no. 1, pp. 1-15, 2016.

[27] J. B. E. Etoa, "New Optimal Pivot Rule for the Simplex Algorithm,” Adv. Pure Math., vol. 06, no. 10, pp. 647-658, 2016.

[28] M. Holzhauser, S. O. Krumke, and C. Thielen, “A network simplex method for the budget-constrained minimum cost flow problem,” Eur. J. Oper. Res., vol. 259, no. 3, pp. 864 872, 2017.

[29] Y. Zhou, Y. Zhou, Q. Luo, and M. Abdel-Basset, “A simplex method-based social spider optimization algorithm for clustering analysis,” Eng. Appl. Artif. Intell., vol. 64, no. September, pp. 67-82, 2017.

[30] S. Voulgaropoulou, N. Samaras, and A. Sifaleras, "Computational complexity of the exterior point simplex algorithm,” Oper. Res., no. June, 2017. 